\title{
Mitophagy and mitochondrial biogenesis in atrial tissue of patients undergoing heart surgery with cardiopulmonary bypass
}

\author{
Allen M. Andres, ${ }^{1}$ Kyle C. Tucker, ${ }^{1}$ Amandine Thomas, ${ }^{1}$ David J.R. Taylor, ${ }^{1}$ David Sengstock, ${ }^{2}$ \\ Salik M. Jahania, ${ }^{2}$ Reza Dabir, ${ }^{2}$ Somayeh Pourpirali, ${ }^{1}$ Jamelle A. Brown, ${ }^{3}$ David G. Westbrook, ${ }^{3}$ \\ Scott W. Ballinger, ${ }^{3}$ Robert M. Mentzer Jr., ${ }^{1}$ and Roberta A. Gottlieb ${ }^{1}$ \\ ${ }^{1}$ Cedars-Sinai Heart Institute, Los Angeles, California, USA. ${ }^{2}$ Beaumont Hospital - Dearborn, Dearborn, Michigan, USA \\ ${ }^{3}$ Department of Pathology, Division of Molecular and Cellular Pathology, University of Alabama, Birmingham, \\ Alabama, USA.
}

\begin{abstract}
Mitophagy occurs during ischemia/reperfusion (I/R) and limits oxidative stress and injury. Mitochondrial turnover was assessed in patients undergoing cardiac surgery involving cardiopulmonary bypass (CPB). Paired biopsies of right atrial appendage before initiation and after weaning from CPB were processed for protein analysis, mitochondrial DNA/nuclear DNA ratio (mtDNA:nucDNA ratio), mtDNA damage, mRNA, and polysome profiling. Mitophagy in the post-CPB samples was evidenced by decreased levels of mitophagy adapters NDP52 and optineurin in whole tissue lysate, decreased Opa1 long form, and translocation of Parkin to the mitochondrial fraction. PCR analysis of mtDNA comparing amplification of short vs. long segments of mtDNA revealed increased damage following cardiac surgery. Surprisingly, a marked increase in several mitochondria-specific protein markers and mtDNA:nucDNA ratio was observed, consistent with increased mitochondrial biogenesis. mRNA analysis suggested that mitochondrial biogenesis was traniscription independent and likely driven by increased translation of existing mRNAs. These findings demonstrate in humans that both mitophagy and mitochondrial biogenesis occur during cardiac surgery involving CPB. We suggest that mitophagy is balanced by mitochondrial biogenesis during I/R stress experienced during surgery. Mitigating mtDNA damage and elucidating mechanisms regulating mitochondrial turnover will lead to interventions to improve outcome after I/R in the setting of heart disease.
\end{abstract}

Authorship note: AMA and KCT contributed equally to this work.

Conflict of interest: The authors have declared that no conflict of interest exists.

Submitted: June 29, 2016 Accepted: January 9, 2017 Published: February 23, 2017

Reference information: JCI Insight. 2017;2(4):e89303. https:// doi.org/10.1172/jci.insight.89303.

\section{Introduction}

Cardioplegia (arresting the heart) is used to protect the heart against ischemic injury during conventional cardiac surgery by reducing myocardial oxygen consumption. Cooling the heart further reduces metabolic demand. Despite the use of cold cardioplegia to protect the heart against ischemia/reperfusion (I/R) injury during conventional cardiac surgery, I/R injury remains a major cause of morbidity and mortality after heart surgery (1). Thus, there is an unmet need to better protect the heart. Efforts to improve upon the current methods of myocardial protection have been stymied, in part, due to the lack of identification of clinically relevant cellular and subcellular targets. To address this conundrum, a better understanding of the beneficial and deleterious processes that characterize the human heart's response to ischemia and reperfusion is needed.

Mitochondria represent a major target of I/R injury; endogenous defense mechanisms must therefore include mitochondrial quality control. An important aspect of this process is mitochondrial turnover, which refers to the integrated processes of degradation via autophagy (mitophagy) and biogenesis. We and others previously demonstrated the importance of cardiac mitophagy in attenuating I/R-induced injury (2-4). A considerable amount of preclinical evidence that mitochondrial biogenesis programs are activated in the setting of cold cardioplegia or I/ $\mathrm{R}$ has been reported (reviewed in ref. 5). However, whether mitophagy and mitochondrial biogenesis occur in the human heart in response to ischemic stress is unknown. The bulk of our knowledge regarding cardiac mitochondrial quality control in the setting of ischemic stress has 


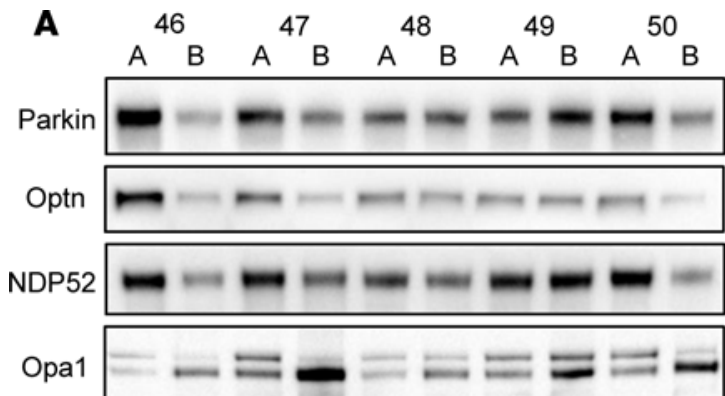

B
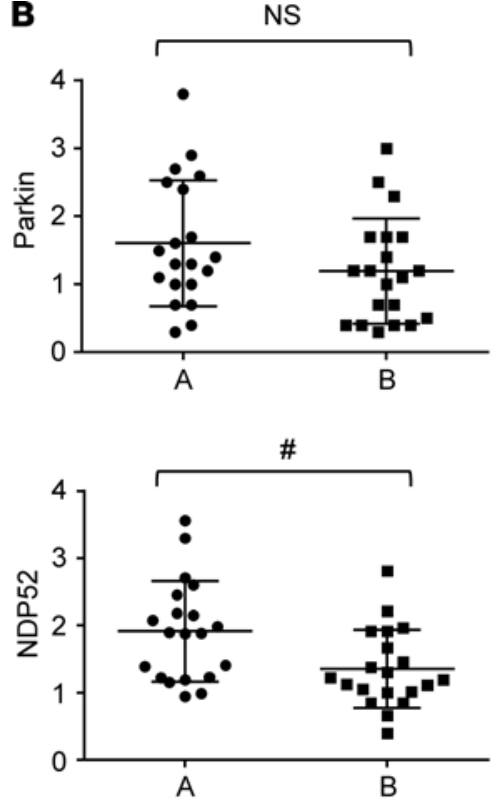

D
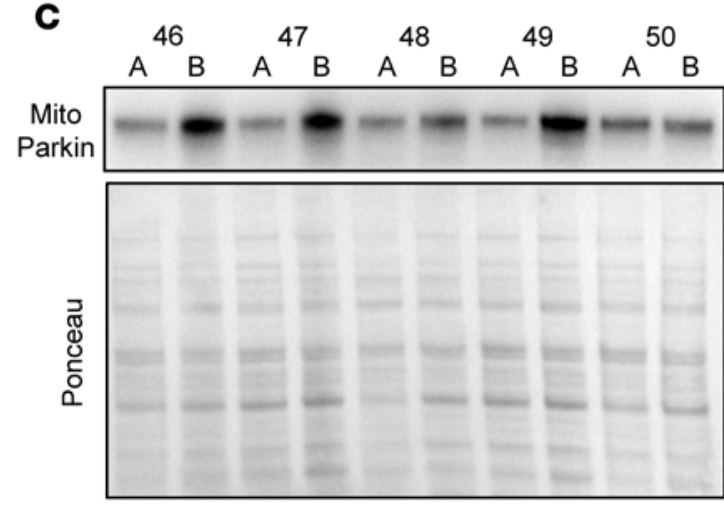

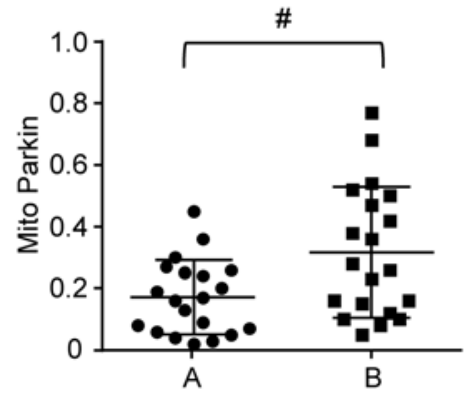

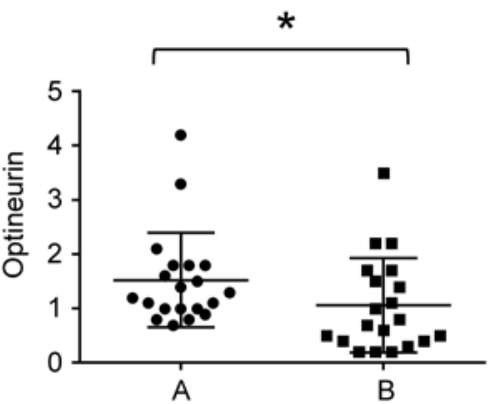

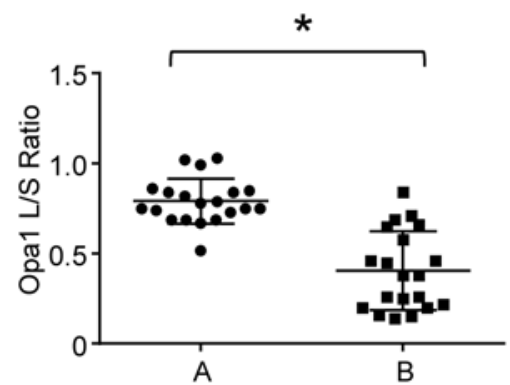

$\mathbf{E}$

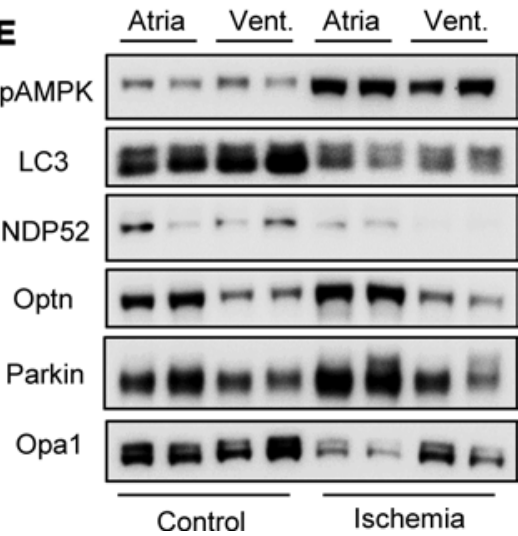

Figure 1. Cardiac surgery triggers mitophagy. Atrial heart biopsies obtained before and after cardiac surgery involving cardiopulmonary bypass (CPB) and cardioplegia from 20 patients were processed to yield whole tissue lysate, as well as crude mitochondrial and cytosolic fractions. (A) Representative Western blot of whole tissue lysate and corresponding Ponceau-stained membrane. (B) Quantitation of $\mathbf{A}$ before (A, solid circles) and after (B, solid squares) CPB. (C and D) Mitochondria-enriched fractions were examined for Parkin translocation as an indicator of mitophagy. (E) Rat hearts were subject to 45 -min global ischemia to compare response between atria and ventricle via Western blot analysis. Data represent mean $\pm \mathrm{SEM}{ }^{*} P<0.05 ;{ }^{\#} P<0.01$. Student $t$ test.

been in cells and animal models. Thus, the purpose of this study was to test the hypothesis that mitochondrial turnover is activated during cardiac surgery. Should this be an active process in the human heart, then mitochondrial quality control mechanisms could be an important new target for the development of more effective clinically relevant myocardial protection strategies.

\section{Results}

We examined biochemical and molecular characteristics of paired atrial biopsies before and after cardiopulmonary bypass (CPB) with cold cardioplegia for coronary arterial bypass graft and/or valve surgery from a cohort of 48 patients (Table 1). To investigate whether mitophagy is triggered during cardiac surgery, we examined Parkin, NDP52, optineurin, and Opa1 by Western blot analysis in 20 patient sample sets. Parkin is an E3 ubiquitin ligase that promotes mitophagy by ubiquitinating outer mitochondrial membrane proteins to facilitate autophagosome recruitment (6). NDP52 and optineurin are recently identified autophagy adapter proteins that facilitate mitophagy in the CNS; however, their roles in the heart have not been described (7). Opa1 resides in the inner membrane of mitochondria and is important for maintaining mitochondrial 
Table 1. Pertinent characteristics of the $\mathbf{4 8}$ patients from which paired samples were analyzed for mitochondrial turnover

\begin{tabular}{|c|c|}
\hline Target & Result $(n=48)$ \\
\hline Age (years \pm SD) & $67.5 \pm 11.6$ \\
\hline \multicolumn{2}{|l|}{ Race } \\
\hline White & $n=44(92 \%)$ \\
\hline Black & $n=2(4 \%)$ \\
\hline Hispanic & $n=1(2 \%)$ \\
\hline Asian & $n=1(2 \%)$ \\
\hline Sex (male) & $n=40(83 \%)$ \\
\hline Body Mass Index $\left(\mathrm{kg} / \mathrm{m}^{2}\right)$ & $31.4 \pm 4.9$ \\
\hline Hypertension & $n=47(98 \%)$ \\
\hline Dyslipidemia & $n=44(92 \%)$ \\
\hline Statin Use & $n=41(85 \%)$ \\
\hline Diabetes & $n=24(48 \%)$ \\
\hline Hemoglobin A1c for entire group & $6.5 \pm 1.6$ \\
\hline \multicolumn{2}{|l|}{ Operation } \\
\hline CABG & $n=26(54 \%)$ \\
\hline Valve & $n=12(25 \%)$ \\
\hline CABG and valve & $n=10(22 \%)$ \\
\hline Cross-clamp time $(\min \pm \mathrm{SD})$ & $93.8 \pm 40.9$ \\
\hline Cardiopulmonary bypass time ( $\min \pm \mathrm{SD})$ & $113.4 \pm 47.4$ \\
\hline
\end{tabular}

fusion; its processing into a short form leads to mitochondrial fragmentation (8), which is a prerequisite for mitophagy (9). Induction of mitophagy was inferred from Parkin translocation to the mitochondrial heavy membrane fraction, depletion of both mitophagy adapters NDP52 and optineurin, and Opa1 processing to its short form in response to the surgery (Figure 1, A-D). Like the depletion of autophagy proteins, which we interpreted to indicate autophagic flux (10), we interpret the combined depletion of optineurin and NDP52, increased Parkin translocation to the mitochondria, and processing of Opa1 to its short form as indications that mitophagy takes place during cardiac surgery. Taken together, these findings provide strong evidence for mitophagy.

These findings are from atrial biopsies; to determine if we might expect the ventricle to show a similar response, we subjected isolated perfused rat hearts to 45 minutes of global no-flow ischemia and compared the ischemic response in atria and ventricles (Figure 1E). AMPK phosphorylation and depletion of microtubule-associated protein 1A/1B-light chain 3 (LC3) were similar in atria and ventricles in response to ischemia, as was processing of Opa1. Optineurin and Parkin were more abundant in atria compared with ventricles. This suggests that similar events were occurring in human ventricles, although we could not sample ventricular tissue in this study.

Myocardial ischemia causes mitochondrial injury, which exacerbates the production of ROS during early reperfusion; mitochondrial ROS are considered a central mediator of injury (11). Mitochondrial ROS are responsible for oxidative damage to mitochondrial DNA (mtDNA). Damaged mtDNA will result in mitochondria with impaired oxidative phosphorylation (OXPHOS) capacity, limiting recovery from cardiac surgery. To investigate whether mtDNA damage occurred, we extracted total DNA from heart biopsies and used short-range and long-range PCR to amplify segments of mtDNA. The short-range PCR serves as normalization factor for mtDNA content, since there is a relatively small probability of encountering a lesion within a short segment of mtDNA, whereas the long-range amplification reaction has a higher probability of encountering a mtDNA lesion that will disrupt amplification. Accordingly, the ratio of long PCR product to short PCR product serves as an indicator of mtDNA damage. We found a consistent decrease in the amount of the long PCR product in the post-CPB samples, indicating that mtDNA damage (comprising single- or double-strand breaks or bulky adducts) had occurred (Supplemental Table 1; supplemental material available online with this article; https://doi.org/10.1172/jci.insight.89303DS1 ).

Induction of mitophagy typically decreases mitochondrial mass. To assess this aspect of mitochondrial turnover, we examined mitochondrial content in whole tissue lysates by Western blot analysis of a representative subunit from each of the 5 OXPHOS complexes. Surprisingly, we observed an increase in mitochondrial mass in post-CPB samples (Figure 2, A and B). Mitochondrial biogenesis involves mtDNA replication and the coordinated synthesis of mitochondrial and nuclear-encoded proteins, which are concurrently assembled into OXPHOS complexes. We examined cytochrome oxidase subunit 4 (CoxIV) as a marker for inner mitochondrial membrane and found a positive correlation with outer mitochondrial membrane marker Tom70 in that the magnitude of increase was similar for Tom70 and CoxIV in each patient (Figure 2C). Consistent with the increase in mitochondrial mass detected via Western blot, we observed an increase in the mtDNA/nuclear DNA ratio (mtDNA:nucDNA ratio) after surgery (Figure 2D). Taken together, our findings provide evidence that synchronized synthesis of mitochondria and replication of mtDNA occur during cardiac surgery, consistent with mitochondrial biogenesis.

To investigate the mechanism by which mitochondrial mass increased, we first examined PGC-1 $\alpha$, the canonical master regulator of mitochondrial biogenesis, and its immediate targets, Tfam and Nrf2. 
A

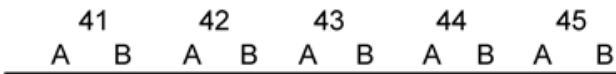

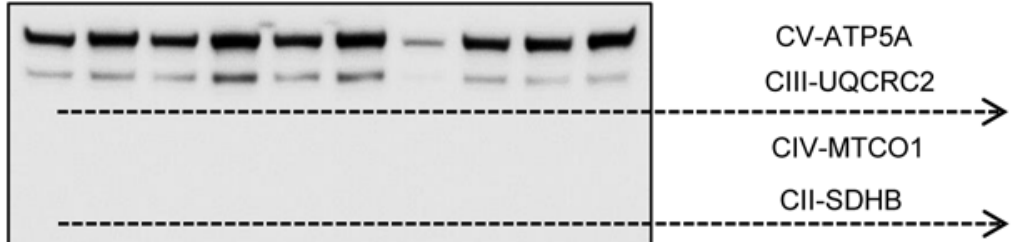

CI-NDUFB8

Exposure 1

B

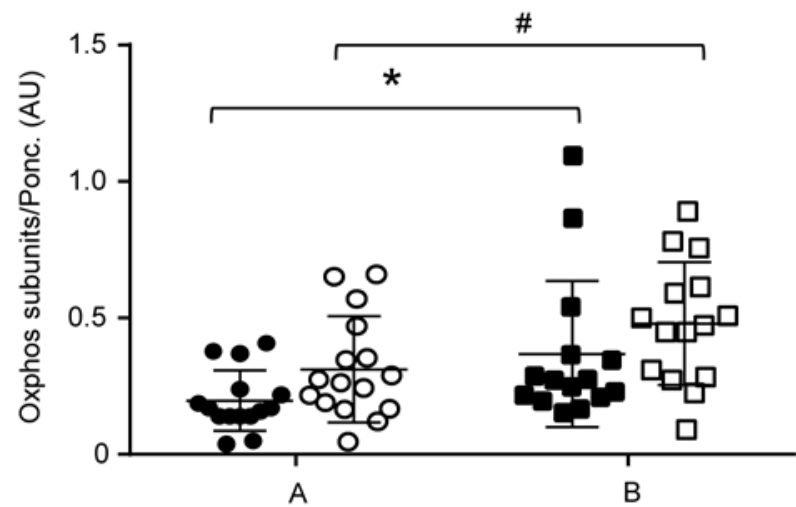

C

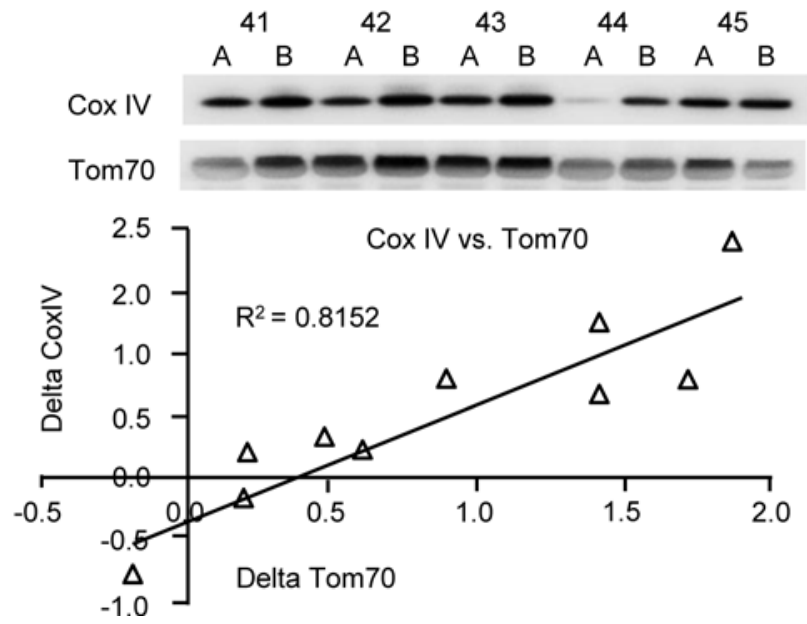

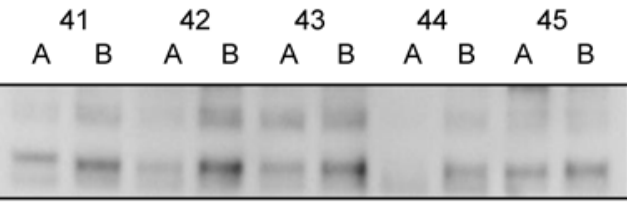

Exposure $2 \circ \square$

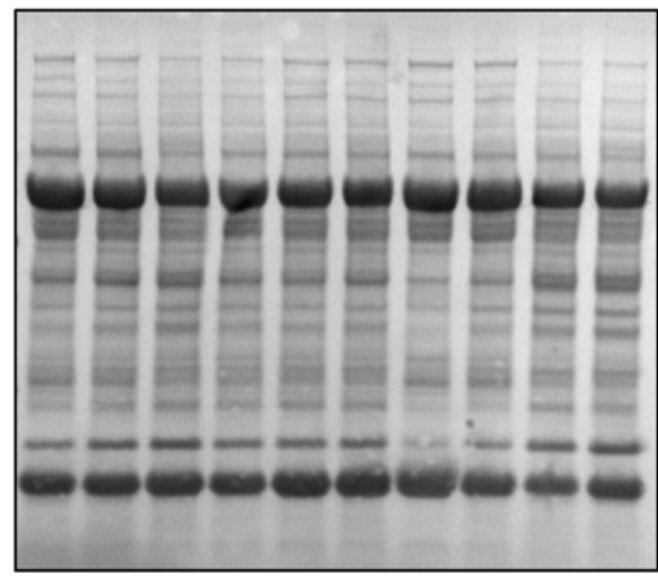

Ponceau
D

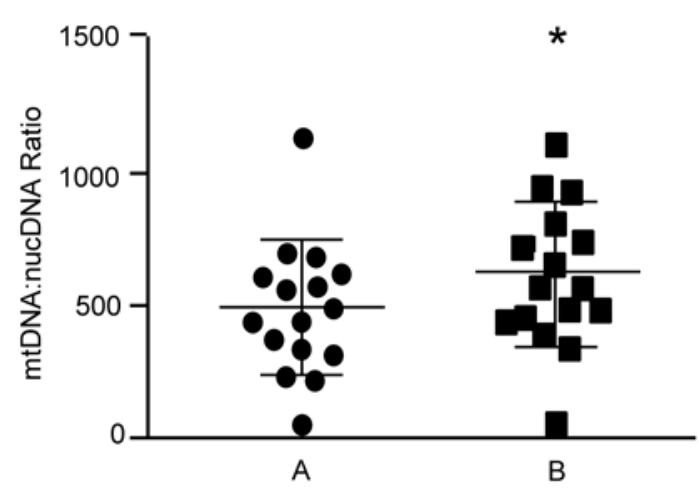

Figure 2. Changes in mitochondrial proteins and mtDNA content during cardiac surgery. (A) Western blot analysis of representative mitochondrial markers in whole tissue lysates from atrial samples before (A, circles) and after (B, squares) cardiac surgery ( $n=20$ patient sets). (B) Quantitation of Western blots, including the representative one shown above. Solid symbols correspond to the densitometric quantitation of lanes from exposure 1 (short exposure), and open symbols correspond to exposure 2 (long exposure). Representative Ponceau-stained membrane used for normalization is shown to right. (C) Representative Western blot of mitochondrial inner (CoxIV) and outer (Tom70) membrane shown with results plotted to examine correlation in response to CPB (10 patient sets plotted). (D) mtDNA:nucDNA ratio was determined by qPCR of mitochondrial and nuclear encoded DNA markers ( $n=16$ patient sets) before (A, solid circles) and after CPB (B, solid squares). Data represent mean \pm SEM. ${ }^{*} P<0.05 ;{ }^{\#} P<0.01$. Student $t$ test $(\mathbf{A}$ and $\mathbf{D})$, linear regression (C).

We found that mRNA for these important regulators did not change from beginning to end of surgery (Figure 3A). We also investigated mRNA for nuclear-encoded mitochondrial proteins including Tom70, cytochrome $c$, and OXPHOS components, and again detected no increase in mRNA after CPB (Figure 3B). These results argued against transcriptional regulation of mitochondrial biogenesis, which in any case would be an inadequate explanation of the increase in mitochondrial proteins in such a short time frame. We next considered the possibility of increased translation of existing mitochondria-targeted mRNAs. For this purpose, we performed polysome profiling to assess the abundance 
A

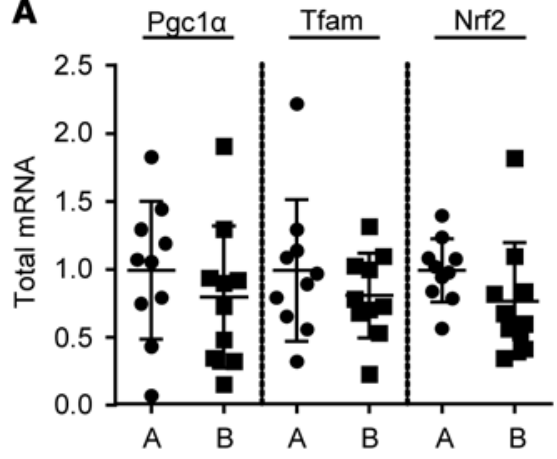

C

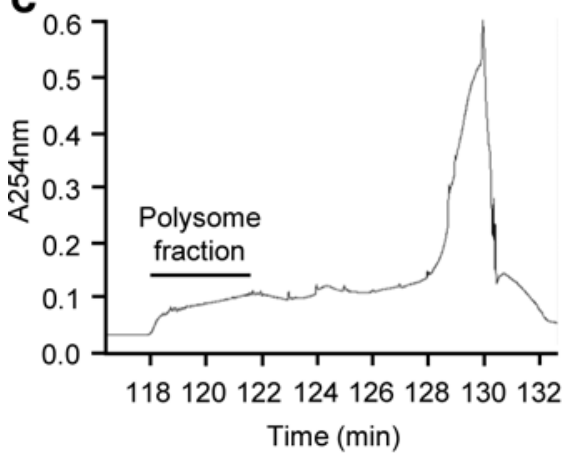

B

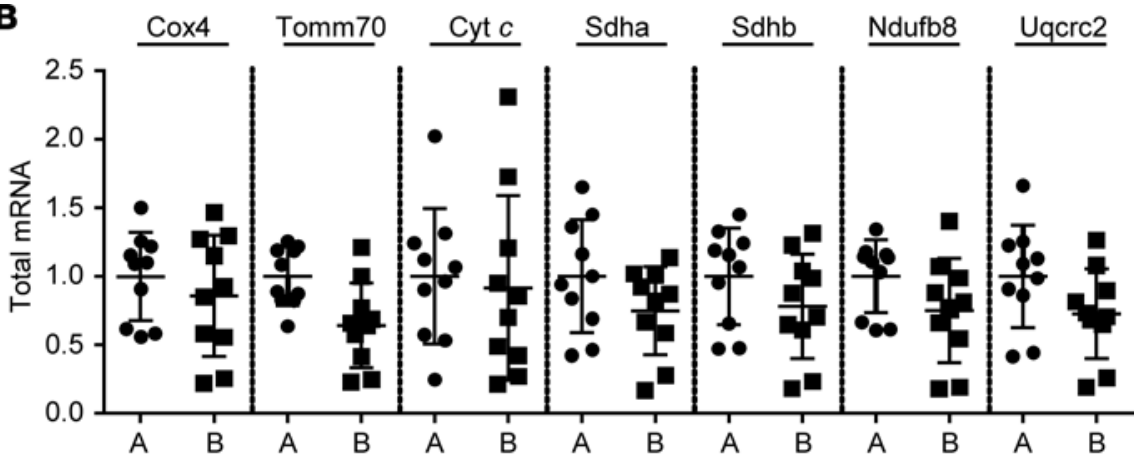

D

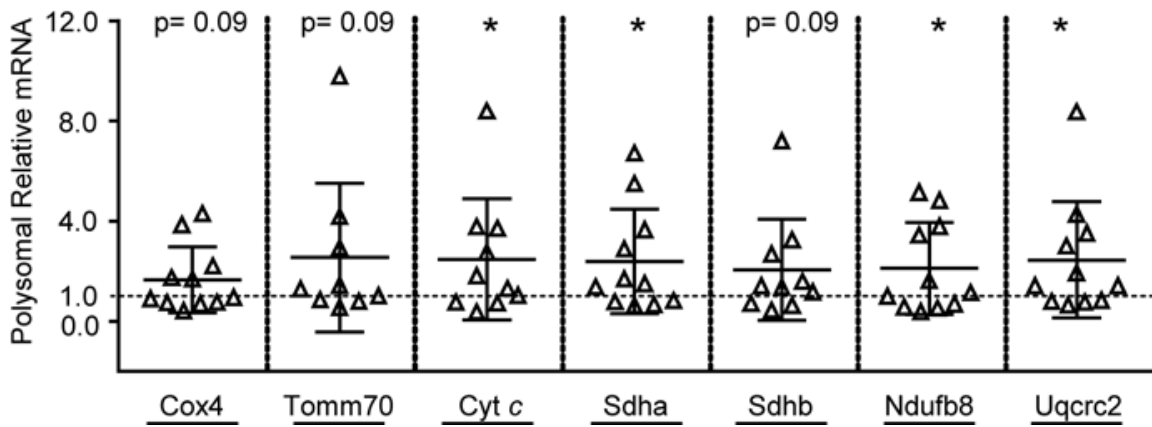

Figure 3. Mitochondrial biogenesis in response to cardiopulmonary bypass is posttranscriptionally controlled. (A) Quantitation of mRNA for transcriptional regulators PGC1 $\alpha$, Tfam, and Nrf2 in pre-CPB (A, solid circles) and post-CPB (B, solid squares) samples. (B) Quantitation of nuclear-encoded mitochondria-targeted mRNAs in pre-CPB (A) and post-CPB (B) samples. No significant differences were found for mRNA targets compared in panel $A$ and B. (C) Example UV optical density profile of sucrose gradient showing distribution of nucleic acid in each fraction (fraction numbers indicated on $x$ axis). (D) Quantitation of mRNA for nuclear-encoded mitochondria-targeted mRNAs in the polysome fraction, where the B value (samples after CPB, open triangles) are plotted as the relative change to its corresponding pair before CPB. Data represent mean \pm SEM. ${ }^{*} P<0.05$ ( $n=11$ patient sets). Student $t$ test.

of relevant nuclear-encoded mitochondria-targeted mRNAs associated with polysomes versus nontranslating fractions. A typical sucrose gradient is shown in Figure 3C. We found that multiple mitochondria-related mRNAs showed redistribution to the polysome fraction in the post-CPB samples, corresponding to the increase in these mitochondrial proteins (Figure 3D). These data reveal translational control as an important mechanism of mitochondrial biogenesis in the setting of myocardial ischemia and reperfusion with cold cardioplegia.

\section{Discussion}

Ischemic preconditioning (12), postconditioning (13), and remote conditioning (14) have been shown to activate cell-autonomous and systemic signaling pathways to protect the heart from I/R injury. We have previously shown that preconditioning induces autophagy and mitophagy essential for cardioprotection (2). We have also shown that autophagy is upregulated in the human heart during cardiac surgery with cold cardioplegia and $\mathrm{CPB}$, suggesting that this cardioprotective pathway may be activated (10). Here, we show that mitophagy and mitochondrial biogenesis are upregulated in the human heart in this setting, raising the possibility that these are part of a coordinated cardioprotective response that we have previously called the homeostatic intracellular repair response $\left(\mathrm{HIR}^{2}\right)(10)$. The present study does not link the upregulation of mitophagy and biogenesis to a reduction in adverse clinical outcomes; additional studies are needed.

Our evidence for mitophagy is based on translocation of Parkin to the mitochondria and processing of Opa1 to its short form, both of which have been linked to induction of mitophagy $(15,16)$. Here, we also monitored levels of NDP52 and optineurin, which are recently identified mitophagy adapters (7, 17-19). To our knowledge, this report is the first to demonstrate their role in ischemic stress response in the human heart. We found that the stress of cardiac surgery leads to decreased levels of these mitophagy adapters, which we interpret as indicative of increased mitophagy similar to our previous findings of decreased p62/ SQSTM1 in the same setting (10). 
The substantial increase in strand breaks in mtDNA is indicative of oxidative stress associated with reperfusion injury. The increase occurs despite ongoing mitophagy, and it is unknown whether mitophagy is able to selectively eliminate mitochondria with the greatest DNA damage or whether it is nonselective. It is also unknown whether the DNA damage is greatest in recently replicated mtDNA, which conceivably could be more vulnerable to free radicals. The study design precluded obtaining tissue at intervals during surgery, limiting our ability to determine the timing of mitochondrial biogenesis with respect to ischemia/cold cardioplegia and reperfusion/rewarming. The finding of perioperative mtDNA damage begs the question whether it will resolve with time, either through enzymatic repair or selective elimination of damaged genomes and expansion of intact copies. It is also unknown whether the magnitude of mtDNA damage in this setting will impact subsequent cardiac function. In a study of heart failure patients, mtDNA damage was increased compared with normal controls when measured at the time of left ventricular assist device (LVAD) placement but was greatly reduced after mechanical unloading, suggesting it is possible to selectively eliminate damaged mitochondrial genomes (20). An alternative explanation is that cells harboring too many damaged mtDNA genomes would undergo apoptosis; the resulting depletion of cardiomyocytes could lead to heart failure. Whether the mtDNA damage sustained during surgery correlates with an increased risk of developing an adverse cardiac event also remains to be determined.

The initial ischemic insult would cause mitochondrial depolarization, leading to Parkin-dependent mitophagy of a subset of the existing mitochondrial population (2), in an effort to restore homeostasis. Previously, we described the $\mathrm{HIR}^{2}$ as an upregulation of autophagy in response to I/R in the context of cardiac surgery. We noted depletion of key autophagy-related proteins, including LC3, Atg5, and p62 (10). While most autophagy proteins are recycled, suppression of protein synthesis during surgical stress limits autophagy until new proteins can be synthesized (21). In the context of cold cardioplegia, we suspect that recycling or replacement of autophagy components is substantially depressed. This is consistent with previous observations that global protein synthesis is suppressed during ischemia (22) - due to hypoxia, nutrient deprivation, and energetic stress on the translational apparatus - and mediated through mTOR and AMPK (23). We were surprised, then, to detect a marked increase in mitochondrial proteins and a parallel increase in mtDNA despite evidence that mitophagy had also transpired. Our findings reveal an increase in translation of preexisting mRNAs in the absence of an increase in mRNA transcripts. The translation of mitochondria-targeted mRNAs may be cap independent, enabling translation despite global suppression of protein synthesis (24). It might seem surprising that adequate protein synthesis could take place within this time frame (average CPB time of 113 minutes, crossclamp time of 94 minutes). A ribosome elongates 20 amino acids per second, so it takes $\sim 20$ minutes to translate 1 mRNA. However, each mRNA can have 8-14 ribosomes, and the mRNA becomes circularized (bridged by protein adaptors) so that the ribosomes are rapidly recycled (25). If the bulk of cap-dependent protein translation is suppressed, the transfer RNAs (tRNAs) needed would be available in higher abundance for translation of mito-targeted mRNAs, further accelerating the rate of translation.

These findings lead us to suggest that mitochondrial biogenesis is linked to mitophagy as a component of the coordinated $\mathrm{HIR}^{2}$ in the setting of I/R stress experienced during cardiac surgery. Further work is needed to relate these findings to patient-specific variables and clinical outcomes. These findings highlight the existence of a robust physiological stimulus for mitochondrial elimination and replacement in response to cardiac surgery and reveal potential pathways for interventions to modulate mitochondrial turnover during or after surgery to enhance ischemia tolerance in patients.

\section{Methods}

Human heart biopsy procurement. Samples of right atrial appendages were obtained from patients undergoing conventional coronary artery bypass grafting (CABG) surgery with $\mathrm{CPB}$ before cross-clamping the aorta and exposure of the heart to a cardioplegic (CP) solution and after short-term reperfusion as previously described (26). First, double purse-string sutures were placed in the atrial appendage and the first tissue was obtained. During placement of the venous cannula, the superior suture was secured to hold the venous cannula in place. The inferior purse string suture remained loose to allow this portion of the atrium to be exposed to blood CP and CPB after removal of the aortic cross-clamp. The second sample of atrial tissue (post-CP/CPB) was then obtained from the area between the purse-string sutures during removal of the venous cannula. 
Table 2. Mitochondria-Related Target Genes (Human)

\begin{tabular}{|c|c|c|c|}
\hline Target & Reference sequence & Forward primer & Reverse primer \\
\hline 185 & X03205 & CTTTCGATGGTAGTCGCCGT & CCTTGGATGTGGTAGCCGTT \\
\hline PGC-1 $1 \alpha$ & NM_013261 & ACCAAACCCACAGAGAACAG & GGGTCAGAGGAAGAGATAAAGTTG \\
\hline Nrf2 & NM_00131390 & TGGGCTCAGCTATGAAAGCA & CCCCAACCAGTTGACAGTGA \\
\hline CoxIV-1 & NM_001861 & GCACTGAAGGAGAAGGAGAAG & ССАСAACССTСTTССАСТС \\
\hline Tomm70 & NM_014820 & ACTACGAGCTACCTTCTACCTG & СATGCTGCСTCTTTTGATCAG \\
\hline SDHB & NM_003000 & AGCGTGAGAAACTGGACGGGCTC & TAGCTGGGGCAGCTGGTGCTA \\
\hline NDUFB8 & NM_005004 & GCTCССTСACСGСTCACAGC & TGCCAGTGCATCGGTTCACCC \\
\hline UQCRC2 & NM_003366 & GGCCAACTACCGTGGAGGTGA & GCACTTCCCGCGACACCACT \\
\hline
\end{tabular}

Preparation of lysates and subcellular fractions. Atrial samples (30-50 mg) were rapidly thawed and subjected to polytron homogenization in ice cold mitochondrial isolation buffer (in mM: sucrose, 250; EDTA, 1; HEPES, 10; $\mathrm{pH}$ 7.4) containing inhibitors (Roche Diagnostics, protease and phosphatase inhibitor cocktails). Nuclei and unbroken cells were eliminated by low-speed spin $\left(1,000 \mathrm{~g}, 4^{\circ} \mathrm{C}, 10 \mathrm{~min}\right)$. A small aliquot of the postnuclear supernatant was reserved (whole lysate [WL]) and the remainder was centrifuged $\left(7,000 \mathrm{~g}, 4^{\circ} \mathrm{C}, 15 \mathrm{~min}\right)$ to obtain the final mitochondria-enriched pellet and supernatant (crude cytosol). The mitochondria-enriched heavy membrane fraction was resuspended in isolation buffer and centrifuged $\left(7,000 \mathrm{~g}, 4^{\circ} \mathrm{C}, 5 \mathrm{~min}\right)$. The pellet was resuspended in ice cold RIPA buffer with inhibitors. Samples were stored at $-80^{\circ} \mathrm{C}$ until use.

Western blot analysis. Total cell lysates, cytosol, and mitochondrial fractions were probed for Rho (catalog sc-373724, Santa Cruz Biotechnology Inc.), Parkin (catalog sc-32282, Santa Cruz Biotechnology Inc.), CoxIV (catalog 4844, Cell Signaling Technology), Tom70 (catalog14528-1-AP, Proteintech) and optineurin (catalog 10837-1-AP, Proteintech), and OXPHOS rodent antibody cocktail (catalog ab110413, Abcam), which recognizes nuclear-encoded subunits Ndufb8 (complex I), Sdhb (complex II), Uqcrc2 (complex III), MTCO1 (complex IV), and ATP5A (complex V). Immunoreactive bands were developed with BioRad Clarity reagent and ChemiDoc XRS system followed by densitometry using NIH ImageJ. Uncropped images of all blots can be found in the online appendix.

$m t D N A$ damage analysis. mtDNA damage in biopsied right atrial appendage from patients undergoing CPB was assessed using quantitative PCR (qPCR) as described previously (27-29). Briefly, total DNA was extracted using the QIAmp DNA Mini Kit (Qiagen) and quantified using the Quant-It PicoGreen dsDNA Quantitation Reagent (Invitrogen). In this assay, the polymerase binds to intact DNA and replicates a fulllength PCR product. In the presence of damaged DNA, however, the polymerase stalls, preventing full replication, thereby resulting in decreased amplification. Almost the entire mitochondrial genome $(16.2 \mathrm{~kb}$ of the $16.5 \mathrm{~kb}$ ) was amplified, yielding the "long-range PCR" amplification product and increasing the probability for detection of mtDNA lesions. This fragment was normalized to mtDNA copy number via a "short-range" PCR amplification product (236 bp) of the mitochondrial genome in which the polymerase has a low probability of having lesions (due to its small amplification size). Data was expressed as the ratio of the average amplification of the long amplification product divided by the short amplification product for each patient and for each group. A decreased long/short ratio is indicative of increased mtDNA damage.

Assessment of mtDNA:nucDNA ratio. Pieces of heart samples (10-15 mg) were used to isolate genomic DNA using a DNeasy Blood and Tissue kit (Qiagen) according to manufacturer's instruction. Primers specific to the human genome were designed using Primer-BLAST online (NIH). Housekeeping nuclear encoded target was GAPDH: Ref. seq - NC_000012.12. For: 5' - TTCAACAGCGACACCCACTC - 3', Rev: 5' - CGCCAGACCCTGCACTTTTT - 3'. Mitochondria encoded target was CO1: Ref. seq - NC_012920. For: 5' - CCTATCCGGAATGCCCCGA, Rev - 5' - CTCCAGGTTTATGGAGGGTTCT - 3'. Equal amounts of total isolated genomic DNA $(0.1 \mu \mathrm{g})$ were used for amplification via qPCR and Sybr Green (Bio-Rad). mtDNA:nucDNA ratios were calculated by normalizing results of mitochondria-encoded gene product against nuclear-encoded gene product. 
Polysome profiling. Human samples were homogenized in a buffer containing $(\mathrm{KCl} 100 \mathrm{mM}$, Tris 20 $\mathrm{mM}, \mathrm{pH} 7.5, \mathrm{MgCl}_{2} 5 \mathrm{mM}, 0.4 \% \mathrm{NP}-40,100 \mu \mathrm{g} / \mathrm{ml}$ cycloheximide and $0.1 \mathrm{U} / \mu 1 \mathrm{RNase}$ inhibitor; Invitrogen). Homogenates were incubated 15 minutes on ice and centrifuged at $18,000 \mathrm{~g}$ for 15 minutes at $4^{\circ} \mathrm{C}$. The supernatants were loaded onto a $15 \%(\mathrm{w} / \mathrm{v})$ to $50 \%$ (w/v) sucrose gradients ([mM] NaCl, $100 ; \mathrm{MgCl}_{2}$, 10; and Tris $\mathrm{HCl}, 10 ; \mathrm{pH} 7.5$ ) and centrifuged at 170,000 $\mathrm{g}$ in a Beckman SW41 Ti rotor for 2 hours at $4^{\circ} \mathrm{C}$. Gradients were fractionated with a BioLogic LP System (Bio-Rad). The polysome fractions were collected, and total RNA was isolated with Trizol (Ambion) following the manufacturer's suggested procedure.

$m R N A$ analysis. Total RNA was isolated from frozen heart samples using a NucleoSpin RNA kit (Macherey-Nagel) according to manufacturer's instruction. qPCR primers were designed and verified using Primer-BLAST online (NIH) (Table 2).

Isolated heart perfusion. Hearts from anesthetized male Sprague Dawley rats from Envigo (225-250 g) were rapidly excised and cannulated onto the Langendorff apparatus and perfused in a retrograde manner with Krebs-Henseleit bicarbonate buffer consisting of $\mathrm{NaCl} 112 \mathrm{mM}$; KCl $5 \mathrm{mM}$; MgSO $41.2 \mathrm{mM} ; \mathrm{KH}_{2} \mathrm{PO}_{4}$ $1.2 \mathrm{mM} ; \mathrm{CaCl}_{2} 1.25 \mathrm{mM}$; $\mathrm{NaHCO}_{3} 25 \mathrm{mM}$; and glucose $11 \mathrm{mM}$. They were gassed with $95 \% \mathrm{O}_{2} / 5 \% \mathrm{CO}_{2}$ ( $\mathrm{pH}$ 7.4). The buffer reservoir height was adjusted to achieve a perfusion pressure of $70-80 \mathrm{~mm} \mathrm{Hg}$, and perfusate temperature was maintained at $37^{\circ} \mathrm{C}$. Hearts were allowed to stabilize for 10 minutes prior to induction of global ischemia via cessation of perfusion for 30 minutes. Temperature was maintained during ischemia by immersing the heart in perfusate maintained at $37^{\circ} \mathrm{C}$. Control hearts were subjected to 30 minutes of normoxic perfusion. At the end of the experiment, atria and ventricles were rapidly excised and immediately snap frozen in liquid nitrogen or further processed for mitochondrial isolation.

Statistics. Values for patient characteristics (Table 1) are expressed as mean \pm SD. All values for Western blot, mRNA, and mtDNA damage analysis are expressed as mean \pm SEM. Two-tailed Student's $t$ test with a $P$ value $<0.05$ was considered statistically significant. Wilcoxon signed rank test was used to analyze mtDNA damage results.

Study approval. All animal studies were performed in accordance with institutional guidelines and approved by the Institutional Animal Care and Use Committee of Cedars-Sinai Medical Center. All studies were conducted under a protocol approved by the Oakwood Hospital IRB and were performed by 2 surgeons.

\section{Author contributions}

AMA and KCT designed, performed, and analyzed most of the experiments and prepared the manuscript. DJRT performed the rat Langendorff heart perfusions and associated Western blots. SMJ and RD performed the cardiac surgeries. DS collected the samples and assisted in data analysis. SP isolated genomic DNA and assisted with mtDNA:nucDNA experiments and developed polysome profiling protocols. AT optimized and executed polysome profiling and mRNA analysis. JAB and DGW performed the long-range vs. short-range PCR to detect levels of mtDNA damage. SWB designed mtDNA damage experiments and assisted in data analysis and interpretation. RAG and RMM were responsible for overall project design, data analysis, and manuscript preparation. All authors reviewed and approved this manuscript.

\section{Acknowledgments}

This study was funded by NIH grants P01 HL112730 (to RAG Program Director and RMM Project Leader), and R01 HL132075 (to RAG). This study was also supported by AHA Scientist Development 15SDG23230013 (to AMA), and R01 HL103859 (to SWB).

Address correspondence to: Roberta A. Gottlieb, Cedars-Sinai Heart Institute A9600, 8700 Beverly Boulevard, Los Angeles, California 90048, USA. Phone: 424.315.2556; E-mail: Roberta.Gottlieb@cshs.org.

1. Cheung CX, Healy DA, Walsh SR. Remote preconditioning and cardiac surgery: regrouping after Remote Ischemic Preconditioning for Heart Surgery (RIPHeart) and Effect of Remote Ischemic Preconditioning on Clinical Outcomes in Patients Undergoing Coronary Artery Bypass Surgery (ERICCA). J Thorac Dis. 2016;8(3):E197-E199.

2. Huang C, Andres AM, Ratliff EP, Hernandez G, Lee P, Gottlieb RA. Preconditioning involves selective mitophagy mediated by Parkin and p62/SQSTM1. PLoS One. 2011;6(6):e20975.

3. Ikeda Y, et al. Endogenous Drp1 mediates mitochondrial autophagy and protects the heart against energy stress. Circ Res. 2015;116(2):264-278.

4. Kubli DA, et al. Parkin protein deficiency exacerbates cardiac injury and reduces survival following myocardial infarction. $J$ Biol 
Chem. 2013;288(2):915-926.

5. McLeod CJ, Pagel I, Sack MN. The mitochondrial biogenesis regulatory program in cardiac adaptation to ischemia--a putative target for therapeutic intervention. Trends Cardiovasc Med. 2005;15(3):118-123.

6. Narendra DP, Youle RJ. Targeting mitochondrial dysfunction: role for PINK1 and Parkin in mitochondrial quality control. Antioxid Redox Signal. 2011;14(10):1929-1938.

7. Lazarou M, et al. The ubiquitin kinase PINK1 recruits autophagy receptors to induce mitophagy. Nature. 2015;524(7565):309-314

8. Wai T, et al. Imbalanced OPA1 processing and mitochondrial fragmentation cause heart failure in mice. Science. 2015;350(6265):aad0116.

9. Twig $\mathrm{G}$, et al. Fission and selective fusion govern mitochondrial segregation and elimination by autophagy. $E M B O J$. 2008;27(2):433-446.

10. Jahania SM, et al. Activation of the homeostatic intracellular repair response during cardiac surgery. J Am Coll Surg. 2013;216(4):719-26; discussion 726.

11. Raedschelders K, Ansley DM, Chen DD. The cellular and molecular origin of reactive oxygen species generation during myocardial ischemia and reperfusion. Pharmacol Ther. 2012;133(2):230-255.

12. Murry CE, Jennings RB, Reimer KA. Preconditioning with ischemia: a delay of lethal cell injury in ischemic myocardium. Circulation. 1986;74(5):1124-1136.

13. Vinten-Johansen J, Zhao ZQ, Zatta AJ, Kin H, Halkos ME, Kerendi F. Postconditioning--A new link in nature's armor against myocardial ischemia-reperfusion injury. Basic Res Cardiol. 2005;100(4):295-310.

14. Hausenloy DJ, Yellon DM. Remote ischaemic preconditioning: underlying mechanisms and clinical application. Cardiovasc Res. 2008;79(3):377-386.

15. MacVicar TD, Lane JD. Impaired OMA1-dependent cleavage of OPA1 and reduced DRP1 fission activity combine to prevent mitophagy in cells that are dependent on oxidative phosphorylation. J Cell Sci. 2014;127(Pt 10):2313-2325.

16. Narendra D, Tanaka A, Suen DF, Youle RJ. Parkin is recruited selectively to impaired mitochondria and promotes their autophagy. J Cell Biol. 2008;183(5):795-803.

17. Heo JM, Ordureau A, Paulo JA, Rinehart J, Harper JW. The PINK1-PARKIN Mitochondrial Ubiquitylation Pathway Drives a Program of OPTN/NDP52 Recruitment and TBK1 Activation to Promote Mitophagy. Mol Cell. 2015;60(1):7-20.

18. Richter B, et al. Phosphorylation of OPTN by TBK1 enhances its binding to Ub chains and promotes selective autophagy of damaged mitochondria. Proc Natl Acad Sci USA. 2016;113(15):4039-4044.

19. Wong YC, Holzbaur EL. Optineurin is an autophagy receptor for damaged mitochondria in parkin-mediated mitophagy that is disrupted by an ALS-linked mutation. Proc Natl Acad Sci USA. 2014;111(42):E4439-E4448.

20. Ahuja P, et al. Divergent mitochondrial biogenesis responses in human cardiomyopathy. Circulation. 2013;127(19):1957-1967.

21. Lawrence BP, Brown WJ. Inhibition of protein synthesis separates autophagic sequestration from the delivery of lysosomal enzymes. J Cell Sci. 1993;105 (Pt 2):473-480.

22. Kao R, Rannels DE, Morgan HE. Effects of anoxia and ischemia on protein synthesis in perfused rat hearts. Circ Res. 1976;38(5 Suppl 1):I124-I130.

23. Magagnin MG, et al. The mTOR target 4E-BP1 contributes to differential protein expression during normoxia and hypoxia through changes in mRNA translation efficiency. Proteomics. 2008;8(5):1019-1028.

24. Komar AA, Hatzoglou M. Cellular IRES-mediated translation: the war of ITAFs in pathophysiological states. Cell Cycle. $2011 ; 10(2): 229-240$.

25. Afonina ZhA, et al. Topology of mRNA chain in isolated eukaryotic double-row polyribosomes. Biochemistry Mosc. 2013;78(5):445-454.

26. Feng J, et al. Altered expression and activation of mitogen-activated protein kinases in diabetic heart during cardioplegic arrest and cardiopulmonary bypass. Surgery. 2013;154(3):436-443

27. Ballinger SW, et al. Mitochondrial integrity and function in atherogenesis. Circulation. 2002;106(5):544-549.

28. Fetterman JL, et al. Mitochondrial DNA damage and vascular function in patients with diabetes mellitus and atherosclerotic cardiovascular disease. Cardiovasc Diabetol. 2016;15:53.

29. Krzywanski DM, et al. Endothelial Cell Bioenergetics and Mitochondrial DNA Damage Differ in Humans Having African or West Eurasian Maternal Ancestry. Circ Cardiovasc Genet. 2016;9(1):26-36. 